\title{
Phénoménologie de l'attention aliénée : Edmund Husserl, Bernhard Waldenfels, Simone Weil
}

Frédéric Moinat

\section{(2) OpenEdition}

1 Journals

Édition électronique

URL : http://journals.openedition.org/alter/1551

DOI : 10.4000/alter.1551

ISSN : 2558-7927

Éditeur :

Association ALTER, Archives Husserl (CNRS-UMR 8547)

\section{Édition imprimée}

Date de publication : 1 octobre 2010

Pagination : 45-58

ISBN : 2-9522374-6-8

ISSN : $1249-8947$

\section{Référence électronique}

Frédéric Moinat, «Phénoménologie de l'attention aliénée : Edmund Husserl, Bernhard Waldenfels, Simone Weil », Alter [En ligne], 18 | 2010, mis en ligne le 01 juin 2020, consulté le 27 juin 2020. URL http://journals.openedition.org/alter/1551 ; DOI : https://doi.org/10.4000/alter.1551 


\section{PHÉNOMÉNOLOGIE DE L'ATTENTION ALIÉNÉE : EDMUND HUSSERL, BERNHARD WALDENFELS, SIMONE WEIL}

Frédéric Moinat

Je me propose ici d'esquisser la phénoménologie d'une attention aliénée, $c^{\prime}$ est-à-dire une attention qui serait rendue étrangère à elle-même. Il importe pour commencer de comprendre ce que signifie pour l'attention d'être rendue étrangère à elle-même. Je tenterai de montrer que l'attention est un mode de la conscience intentionnelle qui apparaît de manière simultanée avec une émergence ou une constitution de sens. Autrement dit, l'attention s'éveille et est maintenue éveillée dans la mesure où elle participe à un dévoilement d'être, à une constitution de sens. Ces deux aspects entrent dans une corrélation noético-noématique : l'attention, du côté de la conscience, est corrélée à un événement survenant du côté du monde. L'acte de se tourner-vers correspond, du côté de l'objet, à un se détacher-de. Exprimé de façon moins technique: nous sommes attentifs lorsqu'il se passe quelque chose, lorsqu'il arrive quelque chose qui est digne de notre attention et qui la nourrit.

Or, l'attention est aliénée dans la mesure où cette corrélation est rompue. Une personne est attentive, voire même vigilante, mais le monde qu'elle vise ne se caractérise pas par une dynamique de constitution de sens; elle est attentive, mais il ne se passe rien, ou plutôt rien d'intéressant, d'important ou de remarquable. Je présenterai des analyses de ce phénomène puisées dans un ouvrage de Bernhard Waldenfels ${ }^{1}$ et dans les textes de Simone Weil sur la condition ouvrière. Je dois toutefois commencer par développer le phénomène de l'attention non aliénée, que l'on peut également appeler attention heureuse. Je me fonderai pour cela sur des analyses qui ont été menées à partir de Husserl et que prend pour thème un collectif sur le phénomène de l'attention paru en 2004 dans la Continental Philosophy Review2.

1. Bernhard Waldenfels, Phänomenologie der Aufmerksamkeit, Suhrkamp Verlag, Frankfurt am Main, 2004.

2. Continental Philosophy Review, 37, Kluwer Academic Publishers, 2004. 


\section{L'attention}

\section{L'attention heureuse}

Natalie Depraz a souligné dans son article publié dans le même collectif ${ }^{3}$ que chez Husserl, l'attention manifeste le caractère actif et libre du sujet qui ne se contente pas de recevoir passivement des impressions plus ou moins fortes mais projette son propre éclairage sur les choses. Et Anthony Steinbock a mis en évidence un autre aspect de la question en montrant que si on considère les analyses husserliennes de la genèse du sens, l'attention n'entre pas seulement en jeu au niveau de l'activité, comme si elle n'était que l'enclenchement d'un projecteur qui rend explicite et thématique ce qui ne l'était pas ${ }^{4}$. Au contraire, l'attention du sujet naît à un niveau passif en étant d'abord éveillée de manière affective. Le sujet est sollicité de la part du monde par l'intermédiaire de forces affectives qui s'exercent sur lui avant même que son attention ne se tourne vers elles. Comme le dit joliment Husserl, l'affection "frappe à la porte du $\mathrm{moi}^{5}$ », c'est-à-dire qu'elle exerce un certain effet sur lui avant même qu'il ne vise par un acte intentionnel exprès la chose qui l'affecte et en prenne clairement et explicitement conscience. Et la façon dont les affections configurent leurs forces relatives, de telle sorte à susciter l'attention du moi, dépend de lois qui ne doivent rien à l'initiative du sujet: ainsi, les effets de contraste, de résonance et de mise en série nourrissent les forces affectives jusqu'au point où le moi leur obéit en se tournant vers elles. Je suis par exemple au volant d'une voiture. Mon attention est dirigée sur la route. À un moment donné, un problème de moteur provoque un bruit bref que je ne remarque pas, restant concentré sur la route. Cela ne veut pas dire que ce bruit n'est rien pour moi ; il m'a affecté, mais trop faiblement pour que mon attention se tourne vers lui. Le même bruit se fait entendre à nouveau. La répétition crée un effet d'insistance qui n'est toutefois pas suffisant pour que je détourne mon attention de la route. Peu après, le même bruit apparaît mais cette fois de manière périodique. Cette mise en série acquiert une force affective suffisante pour s'imposer à ma conscience. Je tourne alors mon attention vers cette série de bruits réalisant du même coup et rétrospectivement que les bruits étaient déjà présents - et la prend pour thème d'une interrogation: d'où ces bruits peuvent-ils venir ? En ai-je déjà entendu de semblables? Est-ce que je risque de tomber en panne avant d'atteindre un garage?

Ce qui m'importe dans cet exemple, c'est le fait que l'éveil de l'attention correspond à une dynamique de constitution de sens. En effet, initialement, l'affection exercée sur le moi correspond à la constitution d'une " objectité »,

3. Ibid., p. 9-10.

4. Ibid. p. 25.

5. Husserliana volume XI Analysen zur passiven Synthesis. Aus Vorlesungs- und Forschungsmanuskripten 1918-1926, édité par M. Fleischer, 1966 (abrégé Hua XI). Traduction française par Bruce Bégout et Jean Kessler avec la collaboration de Natalie Depraz et Marc Richir, De la synthèse passive : logique transcendantale et constitutions originaires, J. Million, Grenoble, 1998. 
c'est-à-dire une sorte de proto-objet, comme le bruit encore indéterminé qui $\mathrm{m}^{\prime}$ affecte alors que je conduis. Lorsque mon attention se tourne vers ce bruit, celui-ci est du même coup constitué en tant que bruit de moteur, il devient un objet, et un objet pertinent dans ma situation présente. Et enfin, à mesure que je le vise et l'examine, il devient un objet qui perdure et qui offre diverses perspectives à mon exploration. Cette constitution de sens où l'initiative ne revient unilatéralement ni au sujet ni au monde, mais aux deux à la fois, Husserl la décrit dans la Synthèse passive comme un « duo constitutif ${ }^{\prime} »$. L'attention est tout à la fois éveillée et éclairante, l'objet est tout à la fois affectant et dévoilé7.

Cette analyse de l'attention heureuse est cependant encore incomplète. En effet, l'analyse de Steinbock n'envisage que le processus d'éveil de l'attention. Elle part de l'affection passive sur un moi inattentif et se termine lorsque l'attention est pleinement éveillée et le moi tourné activement vers l'objet. Or, la question de la continuation de l'attention, de sa durée une fois qu'elle a été pleinement éveillée, est absolument cruciale. Et ceci parce que l'attention a une tendance spontanée, naturelle, à décliner. Il faut ici distinguer entre une cause de distraction externe et interne. Une excitation qui vient distraire l'attention de l'extérieur est un phénomène bien connu 8 . Mais l'attention peut être distraite et décliner alors même que rien ne vient la perturber de l'extérieur, car une distraction interne réside dans l'attention elle-même.

Je vais tenter de rendre compte de cette tendance de l'attention à décliner spontanément en me basant sur une étude pénétrante de Minkowski ${ }^{9}$. Cet auteur aborde le phénomène de l'attention comme une fonction vitale spécifique. Le rôle joué par l'attention consiste selon lui en une médiation nécessaire entre les actes de perception et de pensée d'un côté, et le devenir vital de l'autre. En effet, l'acte de percevoir implique une immobilisation : la perception s'arrête à un objet particulier pour le prendre comme thème. Mais elle entre ainsi en contradiction avec la dynamique vitale qui porte toute vie psychique parce que celle-ci est essentiellement un devenir, un flux en évolution et en changement constants. Autrement dit : «La perception d'un objet immuable, c'est-à-dire celle justement que la psychologie considère comme la plus simple, se trouvait apparemment en contradiction avec le devenir, base essentielle de la vie ${ }^{10}$. » Or, il revient précisément à l'attention d'opérer une médiation entre la perception (ou la pensée) et la vie en

6. Hua XI, p. 52.

7. Ce processus est analysé en détail par A.J. Steinbock dans l'article susmentionné. Steinbock met en évidence sept étapes dans l'éveil de l'attention.

8. Comme le dit finement Aristote : « ... les amateurs de flûte sont incapables de prêter attention aux arguments qu'on leur présente s'ils entendent, serait-ce faiblement, un joueur de flûte, parce qu'ils ont plus de joie à cet art qu'à la présente activité ». Aristote, Ethique à Nicomaque, Flammarion, Paris, 2004, X, 1175b 3-5. Cité par Waldenfels, p. 229.

9. Eugène Minkowski, Vers une cosmologie. Fragments philosophiques, Editions Payot \& Rivages, Paris, 1999, p. 88-96.

10. Ibid. p. 93. 


\section{L'attention}

devenir. D'une part en effet, en réalisant une focalisation intentionnelle sur un objet ou un thème, l'attention permet à la conscience de "s'arrêter à ", $c^{\prime}$ est-à-dire de ne plus passer continuellement d'une perception à l'autre, au gré des affections et des stimulations, pour séjourner auprès d'un certain objet. Mais d'autre part, l'attention doit conserver en elle le devenir de la vie, elle doit rendre possible une focalisation sur un objet qui ne se fige pas en se fixant sur lui, mais qui reste dynamique. L'attention portée à un objet doit ainsi mener une exploration active, découvrir sans cesse de nouveaux aspects, sans quoi elle décline très rapidement. Nous savons bien que lorsque nous fixons quelque chose, une couleur, un son, un paysage ou un tableau sans chercher à l'explorer selon ses multiples facettes, celui-ci s'évanouit. Comme le conclut Minkowski : "Tout en venant rétrécir le champ de conscience, [l'attention] doit constamment, si elle veut progresser et être productive, venir s'alimenter aux sources vives qui l'entourent et qui la baignent ${ }^{11}$. » Nous comprenons à présent pourquoi l'attention a une tendance spontanée à faiblir : en s'arrêtant sur un objet, elle risque à tout moment de s'immobiliser et de perdre ainsi contact avec le dynamisme essentiel de la vie.

Tentons de comprendre plus précisément ce dynamisme essentiel à l'attention en revenant à la dialectique husserlienne entre passivité et activité. On se souvient que Steinbock décrit le processus d'éveil de l'attention comme une transition de la passivité vers l'activité ${ }^{12}$, le passage proprement dit de l'une à l'autre s'effectuant au moment où le moi se tourne vers l'affection. Autrement dit, selon cette interprétation, l'attention commence par être passive pour laisser ensuite la place à l'attention active. Je me rallie ici à la critique que Waldenfels oppose à une telle séparation entre la passivité et l'activité de l'attention ${ }^{13}$. En effet, si nous distinguons nettement d'un côté des forces venant du monde comme l'affection, l'attraction et la répulsion, et de l'autre des forces venant du sujet comme les actes de se tourner vers, de se détourner, examiner, se rapprocher, etc., nous effectuons, fût-ce à notre insu, une réélaboration intellectualiste de l'expérience. Si nous nous tenons à l'expérience elle-même, la passivité et l'activité s'avèrent bien plutôt entrelacées. Le duo constitutif formé par le sujet et le monde est un jeu dans lequel on ne peut pas dire "qui fait quoi », de même que lorsqu'un couple danse, on ne peut pas déterminer la contribution propre de l'un et de l'autre au mouvement de l'ensemble.

Cela ne revient toutefois pas à poser une simple confusion de la passivité et de l'activité. Mais leur distinction nécessaire et éclairante d'un point de vue philosophique n'exclut pas qu'elles soient contaminées l'une par l'autre, intriquées l'une dans l'autre dans l'expérience concrète. Par ailleurs, elles sont configurées dans des polarisations différentes. Parfois, c'est la

11. Ibid. p. 95.

12. Steinbock, op.cit. p. 32

13. Voir en particulier Waldenfels, p. 100sq. 
dimension passive qui domine et le moi se laisse emporter, et parfois c'est la dimension active ${ }^{14}$. De ce point de vue, on peut encore dire avec Steinbock que l'éveil de l'attention comporte un glissement de la passivité vers l'activité. Mais - et ce point est capital pour mon propos - il importe de souligner que la passivité continue à être opérante dans la phase active de l'attention $^{15}$. C'est en effet ainsi que l'on peut comprendre Minkowski quand il dit que l'attention doit être constamment nourrie par les « sources vives » de la vie. Ces sources vives sont les affections passives qui continuent à s'exercer sur le moi, à l'exciter de manière incitative afin qu'il ne relâche pas son attention et poursuive son exploration. Une attention active qui ne fait que prendre le relais d'affections passives va vite s'épuiser dans un activisme tournant à vide. Ainsi, lorsque notre attention a été attirée sur un objet, nous l'examinons avec curiosité et intérêt pendant quelques instants, mais nous n'allons pas commencer à étudier ses différentes faces, comparer ses dimensions avec celles d'autres objets, identifier ses différentes couleurs, etc., si rien ne nous incite à le faire. De même, un exposé ne parvient à capter notre attention jusqu'au bout que s'il dévoile continuellement des choses nouvelles ${ }^{16}$.

Je retiens de ces analyses husserliennes la description de l'éveil et du maintien de l'attention heureuse. L'attention heureuse est une attention vivante; elle est heureuse dans la mesure où elle réussit à conserver le dynamisme de la vie tout en restreignant le champ de conscience sur un objet ou un thème qui perdure. Elle évite ainsi aussi bien une conscience dissipée, ballottée par un chaos de stimuli, qu'une conscience inerte fixée sur un objet. Avant d'aborder l'attention aliénée, il est encore nécessaire de compléter ce premier moment husserlien par la contribution de Waldenfels sur un point capital.

En effet, ces analyses avaient un caractère nettement idéal-typique, au sens où elles se sont basées uniquement sur une relation directe et immédiate entre le sujet et le monde. Rien ne venait s'immiscer dans leur jeu constitutif et l'attention se conformait à la simple alternative suivante: ou une dynamique de constitution de sens maintient l'attention éveillée, ou alors cette dynamique s'essouffle et l'attention décline d'elle-même pour se

14. Cf. ibid., p. 100.

15. Cf. ibid. "Si nous voulons parler ici de passivité, il faudrait alors placer celle-ci dans l'activité elle-même et non dans une phase débutante transitoire, ou une phase terminale en train de s'achever. »

16. Il me semble que l'on peut lire de cette manière les étranges prosopopées de la Synthèse passive qui mettent en scène des objets s'adressant au moi et l'incitant à poursuivre son exploration. Il s'agit bien d'incitations passives qui perdurent alors que le moi accomplit activement ses actes d'observation et d'examen: «L'appel se fait entendre : approche-toi plus près et toujours plus près, considère-moi et fixe-moi alors en changeant ta position, ton regard, etc., tu trouveras en moi-même encore bien du nouveau à voir, toujours de nouvelles colorations partielles, etc., des structures auparavant invisibles du bois que tu n'avais vues auparavant que de manière indéterminée et générale, etc. » (Hua XI, 7) 


\section{L'attention}

dissiper dans une rêverie distraite ou s'éteindre dans le sommeil. Il s'agit à présent de lever cette abstraction en prenant en compte le contexte intersubjectif toujours présent qui médiatise la relation entre le sujet et le monde. Le contexte intersubjectif joue un rôle essentiel notamment dans le phénomène de l'attention à travers les actes de rendre attentif. Lorsqu'un ami me demande: "N'y a-t-il rien ici qui te frappe ? ", il oriente mon attention de telle sorte à me faire voir quelque chose. Et s'il me pose cette question, c'est que je n'étais pas de moi-même réceptif aux sollicitations passives provenant d'une chose qui aurait pu attirer mon regard par des caractéristiques remarquables ou intéressantes. Une fois qu'elle a été guidée en direction de cette chose, mon attention peut prendre le relais et poursuivre son observation en suivant le jeu des sollicitations passives. Il peut aussi arriver que l'invitation à regarder ne me soit d'aucun secours car mon attention ne parvient toujours pas à se porter sur ce qu'il y a à voir. Il arrive également qu'une simple impulsion de départ soit insuffisante pour que je prenne le relais du processus d'attention; l'autre personne va alors accompagner mon attention, la prendre en quelque sorte par la main. Car, comme on l'a vu précédemment, si l'attention ne répond pas aux appels des affections passives, son éveil n'est qu'un feu de paille qui s'éteint très vite. Le soutien que l'autre personne apporte à mon attention est donc une sorte de projecteur qui éclaire ces affections passives pour leur donner plus de force, mais il peut aussi prendre la forme d'une contrainte qui empêche l'attention de se détourner jusqu'à ce qu'elle soit d'elle-même nourrie dans son activité par ce qui est à voir ou à comprendre. Ainsi, le pédagogue qui tente de faire comprendre une démonstration mathématique à son élève doit tenter de le rendre attentif aux éléments du problème qui doivent s'enchaîner pour faire avancer la démonstration, il doit soutenir les synthèses passives qui émanent de ces éléments mêmes et qui montrent le cheminement à suivre. Mais il doit aussi parfois contraindre son élève à garder sa concentration, à maintenir son attention sur la démonstration alors que l'animation de la rue l'attire à travers la fenêtre.

Cet acte de rendre attentif a une importance capitale pour Waldenfels dans la mesure où il nous oblige à mettre en question une conception très courante des rapports intersubjectifs qui, apparemment, va de soi ${ }^{17}$. Cette conception se fonde sur la dichotomie suivante : soit nous entretenons avec les autres des rapports de collaboration, chacun étant l'égal des autres et conservant son autonomie, soit nous entrons dans des rapports dissymétriques de contrainte, où certains s'imposent au détriment des autres, par la manipulation ou la violence. Or, l'acte de rendre attentif n'entre pas dans une telle dichotomie. En effet, lorsqu'une personne attire mon attention sur quelque chose, lorsqu'elle oriente mon regard, elle fait plus que me proposer une chose à voir qu'elle tient pour significative ou importante, elle fait plus que la soumettre à ma libre initiative. Car en me faisant tourner mon regard

17. Voir Waldenfels, p. 233sq. 
dans une certaine direction, elle fait en sorte qu'une certaine configuration de forces affectives puissent $m^{\prime}$ apparaître et, partant, un sens émerger. Elle me met en présence d'un sens déjà en train de se "préconstituer ", un peu comme lorsque je suis mis devant un fait accompli (ou déjà en train de s'accomplir). Autrement dit, elle me fait constituer un certain sens plutôt qu'elle me propose un sens à constituer. Je peux certes résister et ne pas tourner la tête lorsqu'on me dit de le faire, mais dans la mesure où j'obéis, je ne suis pas autonome dans ma constitution de sens. L'autre personne ne cherche pas pour autant à me manipuler au sens où elle $\mathrm{m}$ 'instrumentaliserait en vue de réaliser ses fins propres sans tenir compte des miennes, mais il y a un certain coup de force dans cet acte de rendre attentif parce que l'attention est nécessairement sélective : être attentif ou être rendu attentif à quelque chose, $c^{\prime}$ est du même coup détourner son attention d'autre chose, c'est constituer le monde d'une certaine façon et renoncer à le constituer d'une autre façon. Mais cela va encore plus loin: Waldenfels souligne nettement que, lorsqu'il me rend attentif, autrui contribue à me façonner d'une certaine façon, puisque ce que je vais être, je le constitue par mes constitutions d'objets. Je ne vais pas être le même dans ma vie si, dans mon enfance, les personnes de mon entourage ont habituellement dirigé mon attention vers le monde de la montagne, vers la musique, la misère sociale, ou la littérature ${ }^{18}$. Mais mon monde serait très pauvre si je ne devais compter que sur moi-même pour découvrir tout ce qui est digne d'éveiller mon attention. Ainsi, l'acte de rendre attentif manifeste une telle intrication de sens et de force, de contrainte et de constitution que l'on ne peut pas séparer ces deux aspects ${ }^{19}$. Lorsqu'autrui me rend attentif, il m'ouvre des aspects du monde que je ne remarquais pas, mais il le fait en guidant mon attention, un peu à la façon d'un gouvernail. Or, il arrive que dans l'acte de rendre attentif, l'aspect de la contrainte domine totalement jusqu'à éclipser l'autre aspect de constitution de sens. Cela m'amène enfin à aborder le phénomène de l'attention aliénée.

\section{L'attention aliénée}

Commençons par introduire le concept d'ordre (un concept clé de Waldenfels emprunté à Foucault) en tant que mode de médiatisation intersubjective de l'attention. Tout système de normes qui s'impose à notre expérience pour la réguler d'une certaine manière est un ordre. Ce concept a donc une définition très large qui peut s'appliquer au fonctionnement d'une école, d'une prison ou d'un hôpital, au système judiciaire, à la morale ou encore aux différentes traditions esthétiques. Or, ces ordres institutionnalisés

18. Bien entendu, l'attention peut être dirigée sur plusieurs de ces phénomènes, mais cela ne lui enlève pas son caractère sélectif.

19. Il peut contribuer à réhabiliter des phénomènes d'influence qui, telle la rhétorique, ont mauvaise réputation car on voit y voit une atteinte à l'autonomie et à la souveraineté des sujets. 


\section{L'attention}

déterminent parfois des formes de rendre-attentif dans lesquels l'aspect de la contrainte domine entièrement la constitution de sens.

Soit l'exemple d'un soldat chargé de monter la garde de nuit dans une caserne. Il doit maintenir son attention éveillée pendant plusieurs heures afin de donner l'alarme s'il perçoit des bruits ou des mouvements suspects. On remarque d'emblée combien cette situation est paradoxale par rapport au dynamisme nécessaire de l'attention. Le soldat qui monte efficacement la garde des heures durant alors même qu'il n'y a - comme on dit - «rien à signaler ", parvient en effet à maintenir son attention éveillée sans qu'aucune sollicitation de la part du monde ne vienne la stimuler. Je reviendrai un peu plus tard sur ce paradoxe. Il faut ensuite souligner qu'il n'y a pas ici de duo constitutif dans la mesure où le sujet ne constitue pas du sens en interaction avec des affections passives provenant du monde. En effet, les sollicitations éventuelles (bruit, mouvement) ne sont plus des amorces de sens que le sujet est invité à reprendre, ce ne sont que des signaux déclenchant un comportement prédéterminé (en l'occurrence: donner l'alarme). Le comportement qu'on attend du soldat est entièrement prescrit par l'ordre militaire dont il dépend, et aucune expérience nouvelle, aucune signification inédite, ne tiennent en haleine son attention ${ }^{20}$. En ce sens, l'attention du soldat est aliénée. L'attention aliénée du soldat a un caractère obsessionnel, elle est une forme de surconcentration ${ }^{21}$. Elle se fixe sur les signaux qui seuls comptent pour elle et exerce une sorte de censure envers toute autre affection. Elle se ferme ainsi à toute expérience qui n'entre pas dans l'ordre militaire auquel elle est soumise.

Ce premier exemple m'a permis de relever certains traits caractéristiques de l'attention aliénée. Mais c'est dans les analyses que Simone Weil a menées sur la condition ouvrière que $j$ 'aimerais aborder ce thème plus avant. Le parcours de Simone Weil est bien connu : elle fut professeur de philosophie, militante de la cause ouvrière et, à la fin de sa vie, une philosophe chrétienne d'inspiration mystique. Or, à ma connaissance, elle est la seule philosophe chez qui l'attention est un concept clé, un concept qui joue un rôle de catégorie fondamentale dans sa pensée. Je ne pourrai pas traiter ce thème de manière systématique et exhaustive dans la pensée de Simone Weil ${ }^{22}$, mais il convient de l'aborder de façon générale avant de passer à ses travaux sur la condition ouvrière et les possibilités de son émancipation.

20. Sur l'attention comme fonction de détection et de surveillance, voir notamment Waldenfels, p. 123. Waldenfels ne manque pas de relever que de telles fonctions peuvent être prises en charge par des machines.

21. Cf. Waldenfels, p. 104

22. Au sujet de l'attention chez Simone Weil, voir Joël Janiaud, Simone Weil. L'attention et l'action, PUF, Paris, 2002, et Rolf Kühn, "L'attention comme méthode dé-créative », in : Cahiers Simone Weil, vol. 30, 2007, no 1, p.51-88. Les principaux textes de Simone Weil sur le thème de la condition ouvrière sont édités dans deux livres : La Condition ouvrière, Folio Essais, Gallimard, Paris, 2002 (abrégé CO), et Réflexions sur les causes de la liberté et de l'oppression sociale, Collection Idées, Gallimard, Paris, 1955, ainsi que dans les CEuvres complètes chez Gallimard. 
Pour Simone Weil, la faculté d'attention est sans doute la chose la plus importante dans le développement de l'être humain. En effet, c'est par l'attention que l'homme s'ouvre aux choses mêmes ; mais d'un autre côté, cette ouverture est souvent déficiente ou imparfaite. Loin d'être un donné qui va de soi, l'attention au monde requiert que l'homme ne s'abandonne pas à l'imagination et à la rêverie et fasse l'effort d'adhérer au réel ${ }^{23}$. Ainsi, entre un rêveur dans un champ et un paysan fauchant un champ, Simone Weil valorise l'attitude du paysan car il est beaucoup plus disponible que le premier à la donation de la réalité du champ: "Le bon faucheur a une manière de prendre possession du champ par la pensée. Il connaît mieux le champ que le rêveur qui rêve dans le champ. Le rêveur, en effet, ne sent pas l'existence du monde peser sur lui ; il ne peut distinguer la rêverie de la réalité. Au contraire, pour le bon travailleur, rien n'est plus réel que le champ. La pensée saisit ce qui existe dans le champ. L'attention plane audessus et dirige ${ }^{24}$. » Le problème du rêveur est qu'il néglige le réel alors qu'il s'agit fondamentalement d'être attentif au réel, de percevoir tout ce qui doit être perçu, aussi bien du point de vue de la science (percevoir des structures mathématiques idéelles) que du point de vue des valeurs (percevoir un malheur ou une injustice). D'où cette affirmation lapidaire : "La racine du mal, c'est la rêverie ${ }^{25}$ », ou encore l'importance absolument centrale que Simone Weil attache au développement de la faculté d'attention dans les études ${ }^{26}$.

On voit dans cette brève esquisse du thème de l'attention chez Simone Weil essentiellement une opposition entre l'attention et la distraction. Mais mon propos est de montrer comment le contraste entre l'attention heureuse et l'attention aliénée est élaboré dans la pensée de la philosophe française. Un autre exemple va nous rapprocher de cette thématique. Soit un cycliste débutant ${ }^{27}$. Il est certes très concentré et attentif lorsqu'il s'efforce de tenir son vélo sur la route et de se maintenir lui-même en équilibre. Mais, ce faisant, il manque la véritable attention à l'objet qui caractérise le bon cycliste. En effet, d'une part sa concentration sur la route est crispée. Au lieu de porter son attention de manière globale sur la route en restant ouvert et réceptif à tous les éléments du milieu qui sont pertinents pour sa conduite, le cycliste maladroit se concentre de façon exclusive sur un élément problématique, tel qu'un obstacle à éviter: «il est fasciné par l'obstacle

23. Cette adhésion au réel, qui évoque aussi l'attention à la vie de Bergson, n'empêche pas l'homme d'avoir un espace de jeu, une marge de manœuvre pour son action, bien au contraire, elle est plutôt adéquation au réel.

24. CEuvres complètes, I, p. 386.

25. Lettre au poète Joé Bousquet, in Pensées sans ordre concernant l'amour de Dieu, Gallimard, Paris, 1962, p. 78. Cité par Janiaud, op. cit., p. 5.

26. Voir les «Réflexions sur le bon usage des études scolaires en vue de l'amour de Dieu » in Attente de Dieu, Editions du Vieux Colombier, Paris, 1950, p. 85 et suivantes.

27. CEuvres complètes, I, p. 386. Les citations de l'exemple du cycliste proviennent toutes de cette page. 


\section{L'attention}

comme l'oiseau par le serpent ». Cette tension se manifeste par une rigidité musculaire qui est aux antipodes de la souplesse du bon cycliste. On reconnaît ici le contraste entre une attention vivante qui, comme l'a montré Minkowski, garde un dynamisme et une souplesse internes, et une attention aliénée sous la forme d'une surconcentration obsessionnelle et mécanique, qui présente des analogies avec l'attitude du soldat de garde dont il était question plus haut ${ }^{28}$. De plus, «l'attention du maladroit est une attention aux gestes à éviter. L'attention de l'homme habile est une attention à l'objet ». Il y a donc une réflexivité de l'attention dans le cas du débutant, qui dirige sa conscience sur ses propres gestes au lieu de la diriger sur le monde. On peut parler ici d'une attention mal dirigée, qui s'embarrasse d'elle-même au lieu de s'oublier en faveur du monde et de ses objets. L'attention n'est plus réceptivité et disponibilité au réel, mais fermeture à celui-ci ; ce qui se traduit par un comportement inadéquat à la situation : "L'apprenti cycliste, par exemple, fait attention aux maladresses qu'il peut faire et il les fait. »

$C^{\prime}$ est avec les écrits sur la condition ouvrière que je vais aborder à présent une aliénation beaucoup plus radicale de l'attention que celle que nous venons de voir dans cet exemple, une aliénation qui procède de l'ordre de l'usine dans lequel les ouvriers sont contraints de travailler. Je commencerai par indiquer la ligne de critique que Simone Weil adresse au capitalisme industriel avant de montrer le rôle central qu'y joue l'attention.

Selon Simone Weil, la manière dont Marx a posé le problème de l'aliénation ouvrière est insatisfaisante, ou du moins insuffisante. En effet, en posant que le problème repose essentiellement sur l'accaparement par les capitalistes des profits du travail ouvrier, Marx pensait que la suppression de la propriété privée des moyens de production reviendrait à supprimer la souffrance et l'oppression ouvrières (CO, p. 304-305). Or, c'est avant tout le travail ouvrier dans les usines mécanisées, où le taylorisme est en vigueur, qui est cause de la souffrance ouvrière. C'est la manière de concevoir l'essence du travail ouvrier en usine qui pose problème ; et ce problème ne sera pas résolu par une augmentation de revenu ni par une diminution du temps de travail. Un revenu décent reste une revendication importante, mais Simone Weil ne s'y attarde pas car elle est évidente et souvent trop exclusivement revendiquée, tandis qu'une augmentation des heures de loisir lui semble être un idéal trompeur: «Il ne faut pas tendre à réduire indéfiniment la part du travail dans la vie humaine au profit d'un loisir qui ne satisferait aucune des hautes aspirations de l'homme (comme le pensent ceux qui ont pour idéal deux heures de travail abrutissant, et vingt-deux heures vides d'obligations)... »(CO, p. 257). En effet, le travail est en soi formateur pour l'homme car il peut et doit y trouver un sens. Et le problème

28. À la différence importante que dans le cas du soldat (et, nous le verrons, de l'ouvrier), la situation lui est imposée par l'ordre militaire, alors que le cycliste débutant traverse seulement une phase débutante nécessaire de son apprentissage qu'il surmontera par la suite. Il convient donc de nuancer le caractère d'aliénation dans cet exemple. 
du travail en usine, c'est précisément d'être aliénant pour l'ouvrier, de ne pas lui permettre de s'accomplir : "il s'ensuit que la peur du renvoi et la convoitise des sous doivent cesser d'être les stimulants essentiels qui occupent sans cesse le premier plan dans l'âme des ouvriers, pour agir désormais à leur rang naturel comme stimulants secondaires. D'autres stimulants doivent être au premier plan » (CO, p. 344). On comprend ainsi pourquoi Simone Weil s'attache bien plus à penser un renouvellement radical du mode de travail ouvrier en usine qu'à analyser les rapports socioéconomiques entre les classes ouvrière et capitaliste.

L'aliénation du travail ouvrier résulte de l'application du taylorisme dans les usines. Le travail ne peut pas avoir un sens pour l'ouvrier car « au lieu d'accomplir un travail qualifié, il n'y a plus qu'à exécuter un certain nombre de gestes mécaniques qui se répètent constamment » $(\mathrm{CO}, \mathrm{p} .316)$. L'ouvrier ne peut déployer aucune initiative parce que tout ce qu'on lui demande, $c^{\prime}$ est de se soumettre aux injonctions opératoires de la machine. Il n'a pas de vue d'ensemble du processus de production qui lui permettrait de suivre l'élaboration des objets et de participer ainsi à une tâche commune significative. Pour reprendre la terminologie husserlienne de ma première partie, on peut dire qu'aucune constitution de sens ne s'accomplit ${ }^{29}$. Cette dénonciation du taylorisme est bien connue et en soi assez banale, mais l'analyse de Simone Weil va plus loin. On pourrait en effet croire que le taylorisme est aliénant parce que l'ouvrier serait seulement sollicité à un niveau de comportement corporel et mécanique qui négligerait entièrement le niveau du comportement conscient et réfléchi. L'ouvrier serait ainsi réduit à accomplir un nombre restreint de gestes devenus des quasi-réflexes qui ne mobiliseraient pas sa conscience. C'est ce que semble dire Simone Weil dans un texte de 1936: «la monotonie et plus encore la cadence effroyablement rapide du travail font que [la suite des gestes], toujours extrêmement simple, devient rapidement inconsciente, cristallisée à son tour dans un automatisme physiologique » (CO, p. 258).

Toutefois, la pointe de son analyse est différente, et c'est ce qui fait son intérêt et son originalité. Dans plusieurs autres textes en effet, elle souligne que la souffrance procède précisément du fait que l'ouvrier ne peut pas se contenter d'accomplir des gestes de manière automatique et inconsciente, mais qu'il doit au contraire y mettre toute son attention. Autrement dit, Simone Weil thématise précisément une attention aliénée telle que nous avons déjà pu la voir avec l'exemple du soldat de garde, à savoir le paradoxe d'une attention sollicitée et maintenue en éveil sans être vivifiée. Ce phénomène est paradoxal car, comme nous l'avons vu pour l'attention heureuse, ce sont les stimulations provenant du monde qui nourrissent l'attention en rendant possible une continuation de la constitution du sens. Or, à cause du caractère

29. «L'ouvrier ne sait pas ce qu'il produit, et par suite il n'a pas le sentiment d'avoir produit, mais de s'être épuisé à vide. » (CO, p. 340) 


\section{L'attention}

monotone et mécanique du travail, aucune stimulation ne vient nourrir l'attention de l'ouvrier, qu'il doit pourtant maintenir en éveil.

Selon Simone Weil, cet "attentat contre l'attention des travailleurs » (CO, p. 433) constitue l'essentiel de leurs souffrances en usine. Voyons concrètement pour quelles raisons l'ouvrier est contraint de maintenir son attention éveillée. Premièrement, puisque l'ouvrier est payé à la tâche, il doit absolument éviter de produire des pièces défectueuses qui ne lui seront pas comptabilisées. Il est ainsi amené à surveiller constamment ses gestes, aussi simples soient-ils, car une maladresse peut toujours arriver. Autrement dit, "si le travail parcellaire n'était pas à la tâche, l'ennui qui s'en dégage annihilerait l'attention, occasionnerait une lenteur considérable et des tas de loupés » (CO, p. 52). Deuxièmement, il règne dans l'usine un singulier "mélange de la monotonie et du hasard » (CO, p. 334). L'ouvrier exécute certes à longueur de journée quelques gestes simples toujours identiques, mais cela n'empêche pas des incidents imprévisibles de survenir (une machine se grippe, un outil disparaît, la matière première est de mauvaise qualité, etc.). Or, l'ordre de l'usine attribue aux ouvriers la responsabilité de tous les imprévus, même s'ils n'y peuvent rien. C'est à eux de se débrouiller pour trouver des solutions et relancer la production. "La pensée doit constamment être prête à la fois à suivre le cours monotone des gestes indéfiniment répétés et à trouver en elle-même les ressources pour remédier à l'imprévu. Obligation contradictoire, impossible, épuisante. » (CO, p. 334) Ces derniers mots caractérisent très bien l'attention aliénée: elle est épuisante car contradictoire ; elle tend d'elle-même à l'assoupissement, mais elle est forcée de se maintenir en éveil : "Une légère angoisse empêche cet effet d'assoupissement et force à avoir conscience de la monotonie, bien qu'il soit intolérable d'en avoir conscience ${ }^{30}$. » (CO, p. 334) Nous voyons ici encore un aspect du paradoxe: ce sont les changements et les imprévus survenant de temps en temps qui à la fois rompent la monotonie et en font prendre conscience. Plus précisément dit, c'est en sortant pour de brefs instants de la monotonie que l'on peut précisément en prendre conscience. La mise en veille de l'attention serait bien sûr un profond soulagement; elle permettrait à l'ouvrier de se laisser aller à la rêverie ${ }^{31}$, ou même de porter son attention sur autre chose. Il pourrait ainsi utiliser cette disponibilité d'esprit pour réfléchir, imaginer, etc. On pourrait même aller jusqu'à dire qu'une activité corporelle spontanée et inconsciente est favorable aux

30. Simone Weil résume cette exigence contradictoire dans une formule cartésienne élégante: " [Les ouvriers] sont des choses autant qu'un être humain peut l'être, mais des choses qui n'ont pas licence de perdre conscience, puisqu'il faut toujours pouvoir faire face à l'imprévu » (CO, p. 337).

31. Cf. CO, p. 322 : «En fait, on ne s'accoutume pas [à la monotonie], sauf si l'on peut travailler en pensant à autre chose. Mais alors il faut travailler à un rythme ne réclamant pas trop d'assiduité dans l'attention [demandée] par la nécessité de penser à la cadence du travail. » La rêverie est certes perçue négativement par Simone Weil en tant qu'elle est le contraire de l'attention au réel, mais elle n'a pas le caractère contradictoire et épuisant de l'attention aliénée. 
activités intellectuelles ou artistiques, comme si le corps fonctionnant «tout seul » offrait cette disponibilité à l'esprit. D'où la valorisation de la marche, et des pensées allantes qui l'accompagnent, chez Nietzsche.

Par contraste, l'attention heureuse n'est pas fatigante, même si elle requiert de l'énergie psychique. On peut très bien être intensément actif sans pour autant s'épuiser, puisque nous sommes nourris par cette activité même : "L'attention est un effort, le plus grand des efforts peut-être, mais c'est un effort négatif. Par lui-même il ne comporte pas de fatigue ${ }^{32}$. » De même, dans un roman de George Sand ${ }^{33}$, un médecin pose la question à un enfant : «Ça ne te fatigue pas de faire attention? - Au contraire, ça me repose. » Opposons encore une fois cet enfant « heureux » à celui « à qui on a ordonné d'enfiler des perles pour le faire tenir tranquille » et qui « obéit parce qu'il craint un châtiment ou espère un bonbon » dont parle Simone Weil (CO, p. 344-345) en guise d'analogie avec la situation de l'ouvrier en usine.

Je terminerai cet article en tentant de montrer brièvement ce que deviennent les deux moments de la passivité et de l'activité dans l'attention aliénée. On se rappelle que dans l'attention heureuse, passivité et activité œuvrent de concert pour la constitution du sens: l'attention est d'abord éveillée de manière passive et le sujet prend ensuite le relais pour se tourner activement vers l'objet et mener son exploration sans que les incitations passives s'éteignent pour autant. Dans l'attention aliénée, ces deux moments sont aussi en jeu, mais d'une autre manière : passivité et activité sont dissociées et poussées à l'extrême. Je commencerai par les forces passives. Celles-ci s'exercent sur l'ouvrier de deux manières. Ce sont d'abord les affections provenant de son environnement de travail auxquelles il doit être attentif et répondre d'une manière appropriée. Dès que la machine fait un bruit inhabituel, l'ouvrier doit l'interrompre afin de vérifier que tout est en ordre. Il obéit au bruit comme à un signal qui doit déclencher en lui un certain comportement. Son attention est donc soumise à ses affections passives. En un autre sens, cette soumission concerne l'ordre de l'usine lui-même. L'ouvrier est attentif car il obéit à l'ordre de la production dans lequel il se situe. Il sait qu'on attend de lui qu'il travaille soigneusement et que son salaire en dépendra. Mais d'un autre côté il ne peut maintenir son attention qu'en investissant de manière volontariste une énergie psychique considérable. Autrement dit, il doit compenser le manque de stimulations de la part de son environnement par un considérable effort d'attention. Ce n'est qu'en maintenant son attention éveillée de manière active qu'il peut éviter son déclin. Ainsi, l'attention aliénée se caractérise de manière paradoxale par une extrême passivité d'un côté et une extrême activité de l'autre. Cette dialectique perturbée entre passivité et activité est étroitement liée à une autre dialectique perturbée que Simone Weil thématise explicitement, à savoir

32. Simone Weil, Attente de Dieu, op. cit., p. 92.

33. Le Château de Pictordu. Cité par Bachelard dans Le Rationalisme appliqué, PUF, Paris, 1949, p. 215. 


\section{L'attention}

entre la régularité et la diversité ${ }^{34}$. Dans l'usine taylorisée, la régularité dégénère en "monotonie » et la diversité en "hasard» (cf. CO, op. cit. p. 334). L'attention est écartelée entre un assoupissement dû à la monotonie et une vigilance requise par le hasard. Signalons pour conclure que Simone Weil a tenté de penser quelle pourrait être une technique industrielle qui parvienne à intégrer harmonieusement régularité et diversité et, partant, donner un sens au travail de l'ouvrier. La solution qu'elle entrevoit consiste à remplacer la primauté du mouvement linéaire uniforme (utilisé typiquement dans les chaînes de montage) au profit de « la liaison du mouvement circulaire et du mouvement alternatif »(CO, p. 428). Les dispositifs techniques qui utilisent ces autres mouvements sont plus aptes, selon la philosophe, à stimuler l'attention humaine et sont même chargés d'une symbolique spirituelle. Il y a ainsi une beauté à voir le mouvement droit d'une chute d'eau se convertir avec un moulin à eau en mouvement circulaire; et le mouvement alternatif d'un piston se transmettre aux roues d'un tramway. Simone Weil voyait dans cette beauté technique de quoi nourrir l'attention humaine sans la mettre constamment sur un qui-vive épuisant.

34. Je m'appuie pour ce dernier point sur le livre de Robert Chenavier, Simone Weil. Une philosophie du travail, Les Editions du Cerf, Paris, 2001, p. 559s et 594s. 